\title{
Reversible Phase Transformations during In-Situ Heating of Uncapped $\mathrm{Ge}_{2} \mathrm{Sb}_{2} \mathrm{Te}_{5}$ Films
}

\author{
Chanchal Ghosh ${ }^{1}$, Manish Singh ${ }^{1}$, Paul Kotula ${ }^{2}$, Helena Silva ${ }^{1}$ and C. Barry Carter ${ }^{1}$
}

${ }^{1}$ University of Connecticut, Connecticut, United States, ${ }^{2}$ Sandia National Laboratories, Albuquerque, New Mexico, United States

Chalcogenides-based phase-change materials (PCMs), especially Ge-Sb-Te compounds, show superior properties for non-volatile electronic memory applications (Wuttig \& Yamada, 2007). In operation, the alloy undergoes amorphous-to-crystalline cyclic phase transformations with the thermal energy applied through electrical pulses. In the "Reset" operation, the materials transform from the crystalline phase to the amorphous phase with the application of a short and high amplitude electrical pulse followed by rapid quenching. Whereas, during the "Set" operation, the material transforms to the crystalline phase with the application of a longer and lower amplitude electrical pulse (Friedrich, et al., 2000). These transformations are also associated with the formation of several intermediate structures which can significantly affect their operation. Structural and chemical understanding of these crystallized and recrystallized phases are crucial to design advanced devices with higher capabilities. In-situ heating in TEM (Kooi, et al., 2004) and the subsequent microstructural and microchemical analysis of the nanoscale phase transformation using different TEM techniques is the most viable tool for these analyses (Carter \& Williams, 2016; Williams \& Carter, 2009).

In the present study, the amorphous-crystalline-amorphous transformation has been studied for a Ge2Sb2Te5 (GST-225) specimen with in-situ heating inside TEM (Ghosh, et al., 2020; Singh, et al., 2020a). To study the reversible phase transformation kinetics, in-situ heating experiment is carried out in an uncapped $30 \mathrm{~nm}$ thick GST-225 films on a Si-N supporting film using an Aduro 300 Protochips holder in Tecnai F30 TEM. The video capturing the structural transformation has been recorded in the reciprocal space probing the changes in the selected area diffraction pattern (SADP) during in-situ heating inside TEM. Low-dose HRTEM imaging has been performed in an aberration-corrected Titan ETEM using a direct-electron-detection and large field of view camera (Gatan/Ametek K3-IS). Microchemical analysis of the intermediate phases is carried out using a probe-corrected Titan chemiSTEM. The observations of structures and phase changes are complemented with thermodynamics calculations using Thermo-calc software (Andersson, et al., 2002).

The initial microstructural studies show the island like structure of the film (Tripathi, et al., 2020). The microchemical analysis of the film using electron-energy loss spectroscopy (EELS) confirms the presence of dissolved oxygen along with $\mathrm{Ge}, \mathrm{Sb}$, and Te. Presence of $\mathrm{O}$ is not quite surprising as the film is uncapped and left in the open atmosphere for $\sim 4$ months. The in-situ heating studies shows the crystallization of the film at $\sim 120^{\circ} \mathrm{C}$ with the chemical segregation of $\mathrm{Ge}-\mathrm{O}$ and $\mathrm{Sb}-\mathrm{Te}$ rich regions at the inter-island regions and the islands respectively. Figure 1 displays the snapshots of the SADP and the corresponding bright-field diffraction contrast images at different temperatures during in-situ heating. With further heating at $\sim 200^{\circ} \mathrm{C}$, the grain growth dominates over the nucleation as can be observed at the diffraction pattern with the disappearance of the continuous rings with the emergence of spotty rings. The films amorphized at $\sim 400^{\circ} \mathrm{C}$. The corresponding bright field image at $400^{\circ} \mathrm{C}$ (cf. Figure 1) shows the presence of amorphous grains along with the melted zones. Interestingly, formation of nanoscale recrystallized grains is also observed in the diffraction-contrast images. HRTEM and the subsequent STEM-XEDS analysis of the quenched film at RT confirms these recrystallized grains are rich in Ge. Further STEM-XEDS analysis of the quenched film shows the film is rich in $\mathrm{Ge}, \mathrm{Sb}$ and lean in Te.

Contrary to the general understanding, where the amorphous-to-fcc phase transformation appears at $\sim 150^{\circ} \mathrm{C}$ and the crystalline structure melts at $\sim 600^{\circ} \mathrm{C}$, in the present study, these transformations initiate at a much 
lower temperature. This can be attributed to the presence of dissolved oxygen in the system, which promotes an elemental segregation of $\mathrm{Ge}-\mathrm{O}$ and $\mathrm{Sb}$-Te in the system. Moreover, even though the SAD pattern records the onset of the crystallization at $\sim 120^{\circ} \mathrm{C}$ the low-electron dose HRTEM imaging records the initiation of the earliest stages of crystallization at a much lower temperature (Singh, et al., 2020b). However, the relative volume of the crystallize phases are quite small at that temperature and could not be detected in the SADP.

Acknowledgements: This research is funded by NSF under award DMR-1710468. TEM investigation was carried out at CINT, an Office of Science User Facility operated for the U.S. DOE; Sandia National Labs is managed and operated by National Technology and Engineering Solutions of Sandia, LLC., a wholly owned subsidiary of Honeywell International, Inc., for the U.S. DOE's NNSA contract DE-NA-0003525. The views expressed here do not necessarily represent the views of the U.S. DOE or the U.S. Government.

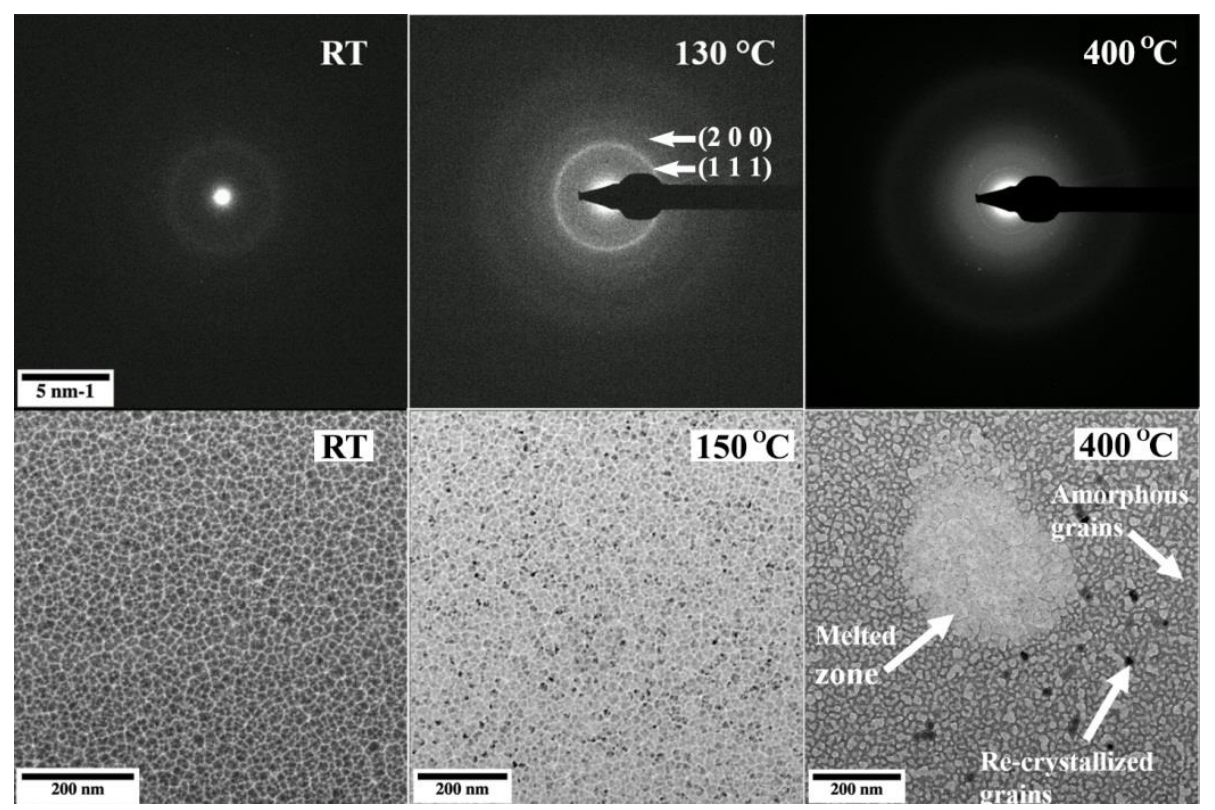

Figure 1. SAD patterns and the corresponding bright-field micrographs from a GST-225 film during in-situ heating inside TEM. Amorphous-crystalline-amorphous transformation of the film is evident from the SAD pattern.

\section{References}

Andersson, J.-O., Helander, T., Höglund, L., Shi, P. \& Sundman, B. (2002). Thermo-Calc \& DICTRA, computational tools for materials science. Calphad 26(2), 273-312.

Carter, C.B. \& Williams, D.B. (2016). Transmission electron microscopy: Diffraction, imaging, and spectrometry. Springer.

Friedrich, I., Weidenhof, V., Njoroge, W., Franz, P. \& Wuttig, M. (2000). Structural transformations of $\mathrm{Ge}_{2} \mathrm{Sb}_{2} \mathrm{Te}_{5}$ films studied by electrical resistance measurements. J Appl Phys 87(9), 4130-4134.

Ghosh, C., Singh, M.K., Watt, J., Silva, H. \& Carter, C.B. (2020). Defect Imaging and Structure Evolution in GST Films During In-situ Heating. Microsc Microanal 26(S2), 1396-1398.

Kooi, B., Groot, W. \& De Hosson, J.T.M. (2004). In situ transmission electron microscopy study of the crystallization of $\mathrm{Ge}_{2} \mathrm{Sb}_{2} \mathrm{Te}_{5}$. J Appl Phys 95(3), 924-932.

Singh, M.K., Ghosh, C., Kotula, P., Watt, J., Silva, H. \& Carter, C.B. (2020a). Direct Observation of Phase Transformations in Ge-Sb-Te Materials. Microsc Microanal 26(S2), 1418-1420. 
Singh, M.K., Ghosh, C., Miller, B., Kotula, P.G., Tripathi, S., Watt, J., Bakan, G., Silva, H. \& Carter, C.B. (2020b). In situ TEM study of crystallization and chemical changes in an oxidized uncapped $\mathrm{Ge}_{2} \mathrm{Sb}_{2} \mathrm{Te}_{5}$ film. J Appl Phys 128(12), 124505.

Tripathi, S., Kotula, P., Singh, M.K., Ghosh, C., Bakan, G., Silva, H. \& Carter, C.B. (2020). Role of Oxygen on Chemical Segregation in Uncapped $\mathrm{Ge}_{2} \mathrm{Sb}_{2} \mathrm{Te}_{5}$ Thin Films on Silicon Nitride. ECS J Solid State Sc 9(5), 054007.

Williams, D.B. \& Carter, C.B. (2009). Transmission electron microscopy: a textbook for materials science. Springer.

Wuttig, M. \& Yamada, N. (2007). Phase-change materials for rewriteable data storage. Nature materials 6(11), 824-832. 